\title{
Anterior Bilateral Dislocation of the Shoulder: Case Report
}

Hicham Douma", Faycal Rifki, Ouahb Azriouil, Mohamed Daoudi, Benabbouha Abdellatif, Khalid Koulali Idrissi

Department of traumatology, Avicenne military hospital, Faculty of medicine and pharmacy of Marrakech, Morocco

DOI: $10.36347 /$ sjmcr.2020.v08i12.007

| Received: 30.11 .2020 | Accepted: 12.12.2020 | Published: 19.12.2020

*Corresponding author: Hicham Douma

Abstract

The anterior bilateral dislocation of the shoulder is a rare clinical entity. We report an observation of a young 22 years old soldier, right-handed person with a history of 2 episodes of antero-internal dislocation of the right shoulder, and who presented to the emergency room for functional impotence of the 2 upper limbs after falling from a ladder of 3 meters. The clinical examination suggesting a bilateral anterior dislocation of the 2 shoulders, confirmed on chest $\mathrm{x}$-ray. An emergency reduction was performed by Kocher's maneuver with success during the first attempt. The patient was scheduled for delayed surgery on his unstable right shoulder.

Keywords: Bilateral, clinical entity soldier dislocation shoulder.

Copyright $\odot 2020$ The Author(s): This is an open-access article distributed under the terms of the Creative Commons Attribution 4.0 International License (CC BY-NC 4.0) which permits unrestricted use, distribution, and reproduction in any medium for non-commercial use provided the original author and source are credited.

\section{INTRODUCTION}

La luxation de l'épaule est une perte des rapports entre la tête de l'humérus et la glène de l'omoplate. Les luxations antéro-internes sont les plus fréquentes. La luxation bilatérale antérieure de l'épaule est une entité rare qui a fait objet de peu de publications (la luxation bilatérale postérieure étant la variété la plus fréquente).le diagnostic est clinique et est confirmé par la radiographie. Nous rapportons une observation $\mathrm{d}$ une luxation antérieure bilatérale chez un jeune militaire de 22 ans.

\section{Patient et Méthodes}

Mr Y.B âgé de 22 ans, militaire droitier, avec comme antécédent 2 épisodes de luxation antéro-interne de l'épaule droite (figure 1), s'est présenté aux urgences pour traumatisme fermé suite a une chute d'une échelle d'une hauteur de 3 mètres. Le patient rapporte qu'il était sur une échelle qui a glissé en avant en entrainant sa chute en arrière avec réception sur les 2 mains, les épaules en abduction et en retro-pulsion; coudes en extension et en supination. A son admission, le patient souffrait de douleur intense des 2 épaules, avec impotence fonctionnelle totale. A l'examen clinique le signe de l'épaulette et le comblement du sillon deltopectoral étaient présents avec une vacuité de la glène à la palpation. La sensibilité et la motricité dans le territoire des nerfs axillaires étaient conservées et le pouls radial bilatéral était présent. Le diagnostic était confirmé sur radiographie du thorax (figure 2). Après avoir expliquer au patient la conduite a tenir, le patient a été sédaté et une réduction a été faite par manœuvre de Kocher (figures 3,4) avec succès dès la première tentative. Apres avoir tester la stabilité, une immobilisation des 2 épaules coude au corps a été faite pendant 3 semaines pour l'épaule gauche et 10 jours pour l'épaule droite.

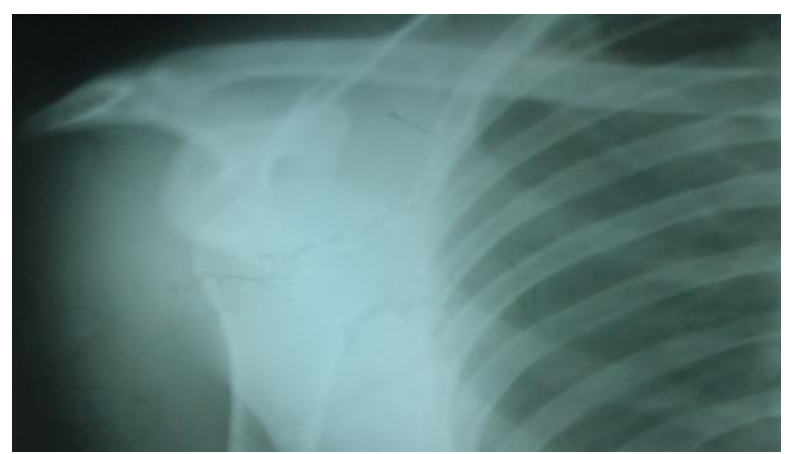

Fig-1: Ancienne radio de l'épaule droite montrant le 1 er épisode de luxation antérieure de l'épaule droite

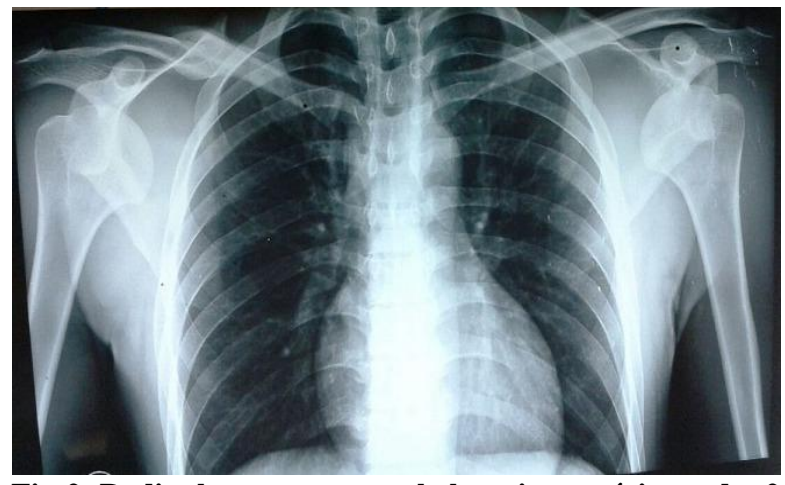

Fig-2: Radio thorax montrant la luxation antérieure des 2 épaules 


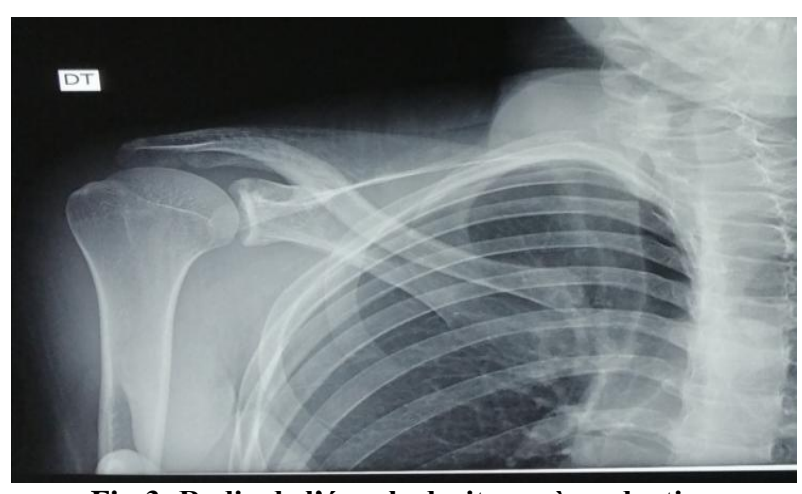

Fig-3: Radio de l'épaule droite après reduction

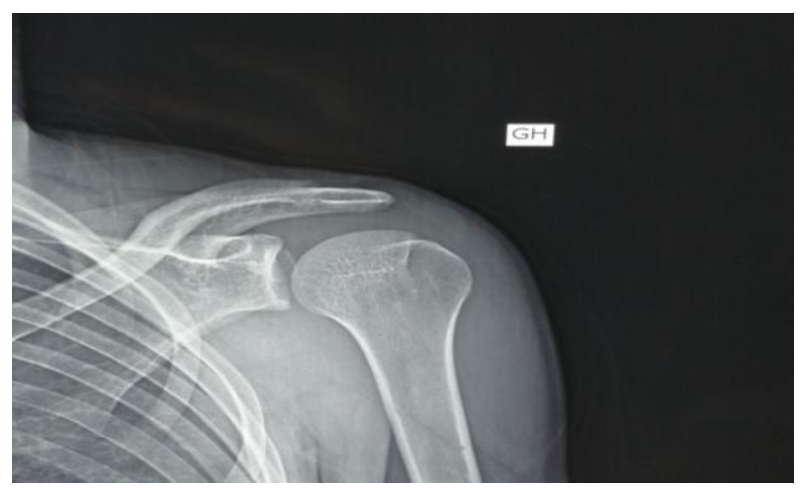

Fig-4: Radio de l'épaule gauche après réduction

\section{DiSCUSSION}

La luxation bilatérale des épaules a été décrite pour la première fois en 1902 suite à des contractions musculaires excessives provoquées par un surdosage au camphre [2]. Elles ont fait l'objet de peu de publications [3, 4]. Brown [5] en 1984 a individualisé, sur une série de 90 cas de luxations bilatérales, trois différentes étiologies: les violentes contractions musculaires (49\%), les traumatismes directs $(23 \%)$ et l'absence de tout traumatisme $(36 \%)$. Ces luxations peuvent être postérieures; variété la plus fréquente $[6,7]$; décrites par Brackstone [1] sous le nom de «triple E syndrome» (l'épilepsie ou toute crise convulsive, l'électrocution et l'extrême traumatisme); antérieures rares avec seulement une trentaine de cas rapportés [6] ou inférieures qui en représentent $0,5 \%$ [7,8].Chez notre patient, le mécanisme était une chute avec réception sur les 2 mains, les épaules en abduction et rétropulsion, les coudes en extension et supination, celle-ci a aggravé l'abduction et la rotation externe des 2 épaules entraînant la perte du contact scapulo-humérale et engendrant donc une luxation antérieure bilatérale. Koufagued et Chafry[12]ont décrit 2 cas de luxations antérieures bilatérales pures .Le 1 er cas a été victime d'AVP ,motocycliste heurte en arrière par une voiture occasionnant chez lui une chute avec réception sur les deux mains projetées en arrière, épaules en abduction et rétro-pulsion, coudes en extension et en supination. Le deuxième cas avait un mécanisme similaire à notre patient, suite à une chute d'une échelle de 2 mètres.
D'autres mécanismes inhabituels ont été décrits. Singh et Kumar [9] ont rapporté un cas ou les deux épaules ont été luxées par des mécanismes différents, chez un patient présentant des antécédents d'instabilité de l'épaule droite. La luxation gauche antérieure était post traumatique secondaire à une chute de moto avec réception directe sur l'épaule alors que le côté droit a été luxé secondairement en antérieur lors du transport, patient tenu par le membre supérieur droit. Bouras et al. [6] ont décrit un cas de luxation antérieure bilatérale des épaules chez un jeune bodybuilder de dix huit ans qui lors d'une séance de musculation, alors qu'il soulevait une barre droite de $40 \mathrm{~kg}$, celle-ci a basculé en arrière provoquant la luxation. Le traitement chez notre patient a été orthopédique par une réduction des luxations sous sédation pour lutter contre la douleur, le stress, l'anxiété et ne pas traumatiser le patient et d'entrainer des lésions supplémentaires par la technique de Kocher qui consiste en une traction avec rotation externe et abduction de l'épaule, puis adduction et rotation interne. . De nombreuses techniques de réduction des luxations sont décrites [10] mais nous adoptons cette dernière car c'est la technique de réduction que nous maîtrisons le plus et elle nous donne entière satisfaction. Quelque soit la manœuvre de réduction utilisée, elle doit être douce et progressive afin de ne pas aggraver les lésions [11]. La chirurgie a été envisagée par technique de butée antérieure de Latarjet afin de remédier a l'instabilité de l'épaule droite du patient.

\section{CONCLUSION}

La luxation antérieure bilatérale est une entité clinique rare, Les étiologies de luxations bilatérales antérieures rapportées sont tous post traumatique alors qu'elles sont non traumatiques dans les luxations postérieures et dues souvent à des contractions musculaires excessives. Le caractère bilatéral ne doit pas changer la prise en charge.

\section{Conflits d'intérêt} d'intérêts

Les auteurs ne déclarent aucun conflit

\section{REMERCIEMENTS}

Je tiens à remercier tous ceux qui, par leurs coopérations, leurs suggestions et leurs corrections judicieuses, ont contribué à l'élaboration de cet article.

\section{REFERENCE}

1. Brackstone M, Patterson SD, Kertesz A. Triple "E" syndrome: bilateral locked posterior fracture dislocation of the shoulders. Neurology. 2001 May 22;56(10):1403-4.

2. Myenter H. Subacromial dislocation from muscular spasm. Ann Surg. 1902 Jul;36(1):117-9. 
3. Dunlop CC. Bilateral anterior shoulder dislocation: a case report and review of the literature. Acta Orthop Belg. 2002 Apr;68(2):168-70.

4. Devalia KL, Peter VK. Bilateral post-traumatic anterior shoulder dislocation. J Postgrad Med. 2005 Jan-Mar;51(1):72-3.

5. Brown RJ. Bilateral dislocation of the shoulders. Injury. 1984 Jan;15(4):267-73.

6. Bouras Y, Elandaloussi Y, Nadil MA. Luxation antérieure bilatérale des épaules chez le bodybuilder (à propos d'un cas) Journal de Traumatologie du Sport. 2009 Dec;26(4):247-249.

7. Ryan J, Whitten M. Bilateral locked posterior shoulder dislocation in a footballer. Br J Sports Med. 1997 Mar;31(1):74-5.

8. Petty K, Price J, Kharasch M. Bilateral Luxatio Erecta: a Case Report. J Emerg med. 2014 Feb;46(2):176-179.
9. Singh S, Sandeep K, Kumar R, Sudhir M. Bilateral anterior shoulder dislocation: a case report. Eur J Emerg med. 2005 Feb;12(1):33-5.

10. Genin J. Prise en charge de la luxation glénohumérale par les médecins de stations de sports d'hiver. Journal de Traumatologie du Sport. 2001 Mar;18(3):113-122.

11. Sirveaux F, Molé D, Walch G. Encycl Méd Chir, Appareil locomoteur. Paris: Editions Scientifiques et Médicales Elsevier SAS:20; 2002. Instabilités et luxations glénohumérales.

12. Koufagued K, Chafry B, Bouabid S, Chagar B. Les luxations bilatérales antérieures pures des épaules à mécanisme particulier: à propos de deux cas. Pan African Medical Journal. 2015;22(1). 